\title{
Pupillometry in Infancy Research
}

\author{
Robert Hepach ${ }^{1} \&$ Gert Westermann ${ }^{2}$ \\ ${ }^{1}$ Max Planck Institute for Evolutionary Anthropology, Leipzig \\ ${ }^{2}$ Department of Psychology, University of Lancaster
}

Corresponding authors:

Robert Hepach

Department of Developmental and Comparative Psychology

Max Planck Institute for Evolutionary Anthropology

Deutscher Platz 6

04103 Leipzig

Germany

$+49-341-3550-484$

hepach@eva.mpg.de

Gert Westermann

Department of Psychology

Lancaster University

Lancaster LA1 4YF

United Kingdom

+44 (0)1524592942

g.westermann@lancaster.ac.uk 


\begin{abstract}
The human pupil is a small opening in each eye that dilates in response not only to changes in luminance but also to novel events. This makes changes in pupil diameter an attractive measure in studies on infants' and young children's physical and social cognition. However, designing and interpreting pupillometry studies for developmental populations comes with its own caveats. Here we give an overview of how psychologically induced changes in pupil diameter have been investigated and interpreted in developmental studies. We highlight the methodological challenges when designing experiments for infants and young children and provide several suggestions to address common problems. The fact that pupillometry provides a sensitive measure of the time course of responses to novelty extends the scope of possibilities for researchers studying infant cognition and development.
\end{abstract}

Keywords: methods, infancy, childhood, development, eye tracking, pupillometry, pupil dilation 


\section{Pupillometry in Infancy Research}

Everything that infants see traverses through the pupil, a small circular opening in the irises of each eye. Unbeknownst to the infants, and to the majority of adults, the pupil not only regulates the influx of light but also constantly oscillates in response to activity of the autonomous nervous system resulting from psycho-sensory stimulation (Loewenfeld, 1958). In adults, increases in pupil diameter have been documented as a response to emotional stimuli, to cognitively demanding tasks and, in general, in situations of increased attention to novel and stimulating information (Goldwater, 1972; Laeng, Sirois, \& Gredebäck, 2012; Sirois \& Brisson, 2014). These findings make pupil dilation a promising candidate measure of cognitive processing also in preverbal or just-verbal populations to supplement more established measures such as looking times (e.g., Cashon \& Cohen, 2000; Cohen, DeLoache, \& Pearl, 1977; Fantz, 1963, 1964; Woodward, 1998). Given the wide use of eye tracking in infancy research (Aslin, 2007; Gredebäck, Johnson, \& von Hofsten, 2009; Oakes, 2012) and the fact that modern eye trackers measure pupil size between 30 and 500 times per second it is therefore surprising that only a handful of studies so far have made use of this measure in research with infants (see Fig. 1 for an illustration).

In some cases results from pupil dilation match those from looking time studies. For example, in violation-of-expectation (VOE) paradigms that present infants with a novel, unexpected event, both looking time and pupil dilation increase to the novel stimulus while participants are in a psychological state of increased attention (Jackson \& Sirois, 2009). However, there are also important differences between looking time and pupil size measures. First, measures of looking time are cumulative and different mechanisms of allocating attention may result in the same overall global looking time (Aslin, 2007). In contrast, pupil dilation measures provide 
a continuous variable across the whole stimulus presentation that allow for analysis of the time course of information processing (see Bradley, Miccoli, Escrig, \& Lang, 2008; Kahneman, Tursky, Shapiro, \& Crider, 1969; Libby, Lacey, \& Lacey, 1973, for a direct comparison of physiological variables). Second, in comparison to global looking time, pupil dilation does not suffer from a diminishing response over trials when participants become tired and less attentive because it does not require them to look at a stimulus for more than a few seconds (Jackson \& Sirois, 2009; see also Sirois \& Jackson, 2012, for a detailed discussion). And third, comparisons of looking times between younger and older age groups are difficult because with increasing age, humans become more able to voluntarily control their eye movements and increases in looking time may therefore be driven by different processes in infants and adults. This problem does not exist for pupil dilation: changes in pupil size have a physiological basis rooted in activation of the subcortical structure locus coeruleus (Laeng, Sirois, \& Gredebäck, 2012; Nieuwenhuis, De Geus, \& Aston-Jones, 2011) and are an unbiased involuntary marker of nervous system activity. The positive relation between increased attention and pupil diameter has been validated in numerous studies with adults (Goldwater, 1972, Laeng et al., 2012, Sirois \& Brisson, 2014) in ways that measures of looking time have not.

Following suggestions by Aslin (2007) to seek out alternatives to measures to global looking time, assessing changes in pupil size in response to novelty has the potential to clarify and extend findings regarding infants' and young children's understanding of the physical and social world. Importantly, unlike looking times pupil dilation is not interpreted with regard to the duration of a response but by the magnitude of physiological activity triggered by an experimental manipulation. As such it bears more resemblance to event-related potentials (ERP) than measures of 
looking time. What makes pupillometry particularly interesting for developmental psychologists is the increased flexibility it offers in designing experiments. Because it is not cumulative data do not have to be collected on a still image until the subject looks away from the presentation screen. Instead, the dynamic nature of pupillary changes allows for the presentation of video or live stimuli where subjects can remain engaged while changes in attention are recorded over time intervals as brief as 3 to 10

s.

Pupillometry in infancy and toddlerhood is a relatively young research field, and similarly to eye tracking (Oakes, 2012) and event related potentials (de Haan, 2013), studies with preverbal or just-verbal populations come with their own caveats. In the rest of this paper we provide a brief overview of the measure of pupil dilation before focusing on its application in infants and toddlers with some remarks on how best to use it in developmental populations. In comparison to previous reviews and methodological papers (Goldwater, 1972; Hepach, Vaish, \& Tomasello, 2015; Laeng et al., 2012; Nyström, Falck-Ytter, \& Gredebäck, 2015; Sirois \& Brisson, 2014) we (1) focus exclusively on infancy research, (2) review past research and analysis strategies, (3) provide a theoretical framework for interpreting changes in pupil diameter in infants, and (4) address the particular methodological challenges that arise specifically in infancy research. Our aim is to highlight the benefits of using pupil dilation for researchers studying infant and toddler cognition, and to suggest guidelines for experimental designs using this measure.

\section{Pupil Dilation in a Nutshell}

The human pupil responds first and foremost to light. An increase in luminance will lead to pupillary constriction, and a decrease to pupil dilation. Nevertheless, even under constant light conditions the pupils continuously oscillate, 
increasing and decreasing in size. At any given point in time pupillary changes represent a state of homeostasis between the working of parasympathetic activity constricting and sympathetic activity dilating the pupil (Wilhelm, 1991). An example of the typical time course of pupillary change upon presentation of a stimulus is shown in Figure 2. The pupillary light reflex (PLR) characterizes the initial pupillary constriction (PC) in response to a bright stimulus appearing on a dark background. This initial constriction is always followed by a subsequent re-dilation of the pupils (PR). PC and PR together usually last approximately $5 \mathrm{~s}$ (see Fig. 2B). If the luminance remains constant, the pupils will then oscillate (PO) until a change in luminance will again induce a PLR.

Pupillary changes following psycho-sensory stimulation are commonly categorized into tonic state and phasic changes (Granholm \& Steinhauer, 2004). Recording pupil size over a period of several seconds or minutes and computing the average size during that time reveals the tonic state of pupil dilation. Experiencing a psychologically taxing event during that time period would lead to an increase in average pupil size. Tonic state therefore measures a kind of baseline arousal state of the participant and can be useful for measuring the effect of an experimental manipulation over longer periods of time (Hepach et al., 2012; see Fig. 2D \& 2F).

However, more commonly researchers focus on the rapid phasic changes in pupil dilation that occur as an immediate effect in response to a stimulus (see Fig. 2C). These measures are time locked to the onset of the stimulus event (at time $x$ ) and are measured during a pre-defined time interval $x_{t}$ following the onset of this event. Beatty and Lucero-Wagner (2000) refer to phasic changes as a task-evoked pupillary response (TEPR) comparable to ERPs and describe them as indicators of underlying neural activity. At different parts of the pupillary curve the effect of such 
psychologically induced pupillary changes varies. Given that pupil dilation over time is volatile even within the same participant and using the same stimulus and time window, the mean pupil size within each window $x_{t}$ may differ between experimental trials. Adult experiments use several trials to average out these variations (e.g., Bradley et al., 2008).

There are several common misconceptions about pupil dilation. First, the current empirical evidence suggests that there is no psychologically induced pupillary constriction but only dilation. Claims that pupil size may vary in a bidirectional manner with the degree of pleasantness or interest value have been met with scepticism and caution (Loewenfeld, 1993). Second, pupil dilation does not change as a function of stimulus valence. Positive and negative pictures or sounds alike result in the same degree of pupil dilation if they excite sufficient arousal (Bradley et al., 2008, Partala \& Suraka, 2003). Third, the pupil does not change with the degree of likability of a stimulus and knowing something about the size or change in size of a participant's pupil does not reveal any one specific psychological state (Goldwater, 1972; Loewenfeld, 1993; Lowenstein, 1920; Sirois \& Brisson, 2014). Rather increased pupil dilation reveals psychological engagement in different psychological states and systematic changes in pupil size always have to be interpreted in comparison to experimental control conditions just like any other physiological variable.

\section{Research with Pupil Dilation in Adults}

Pupil dilation is a well-established measure of sympathetic activity in adults and for the present purposes we will only briefly highlight some key findings (see Goldwater, 1972; Laeng, Sirois, \& Gredebäck, 2012, Sirois \& Brisson, 2014, for extensive reviews). Different underlying mechanisms for changes in pupil size have 
been recognized. First and foremost, but of least interest in our context, is the change in pupil diameter due to changes in luminance. Parasympathetic nerve fibers regulate the influx of light onto the retina by gradually dilating the pupil in darker environment and constricting it when overall luminance is high (Loewenfeld, 1993). Second, the diameter of the pupil increases with a participant's experienced internal arousal that can, for example, be induced through viewing or listening to emotionally charged stimuli (Bradley et al., 2008; Hess \& Polt, 1960; Partala \& Surakka, 2003). Third, the human pupil dilates as a function of mental effort, e.g., while performing basic arithmetic (Bradshaw, 1967) and memorizing numbers of varying digits (Kahneman \& Beatty, 1966). Similarly, changes in pupil dilation have been studied as a kind of cognitive index to measure mental workload (Marshall, 2002). Participants can therefore voluntarily assume psychological states that will result in increased pupil size, although it is questionable whether the specific onset and degree of pupil dilation can be controlled in this manner. Fourth, pupil dilation is influenced by so-called top down processes. Subjects who are guessing the outcome of a stimulus sequence show greater pupil dilation than when they are told the sequence in advance (Hakerem, 1974). Similarly, imagining a sunny sky compared to a nigh sky constricts adults' pupils significantly (Laeng \& Sulutvedt, 2014). Fifth, and importantly for infancy research, pupil dilation increases with the novelty and motivational significance of a stimulus (Nieuwenhuis, De Geus, \& Aston-Jones, 2011) and has been used as a measure of 'surprise' in adults (Preuschoff, 't Hart, \& Einhäuser, 2011). Increases in pupil dilation as well as increased looking time are part of a physiological orienting responses toward a novel and significant stimuli (Sokolov, 1963). This makes measures of pupil dilation highly promising for studying infant cognition. 
The field of pupillometry in infancy and early childhood is relatively new. However, the wide array of applications of the measure ranging from physical cognition (Jackson \& Sirois, 2009; Sirois \& Jackson, 2012), social cognition (Gredebäck \& Melinder, 2010, 2011; Hochmann \& Papeo, 2014), emotion perception (Geangu, Hauf, Bhardwaj, \& Bentz, 2011; Hepach \& Westermann, 2013), motivation (Hepach, Vaish, \& Tomasello, 2012, 2013), and application in clinical populations and their caregivers (e.g., Anderson, Colombo, \& Shaddy, 2006; Falck-Ytter, 2008; Martineau, Hernandez, Hiebel, Roché, Metzger, \& Bonnet-Brilhault, 2011) suggests a potential that is slowly but steadily emerging (see Table 1 for a more exhaustive list of recent publications). Jackson and Sirois (2009) assessed both pupil dilation and looking time in studies on object permanence with 8- and 10-month-old infants and found increased pupil dilation in response to unexpected physical events (Jackson \& Sirois, 2009; Sirois \& Jackson, 2011). Gredebäck and Melinder (2010) combined measures of anticipatory looking and pupil dilation and found that 12-month-old but not 6-month-old infants anticipated the correct trajectory of an observed feeding action. Corresponding to this result, only the older infants showed greater pupil dilation when observing a scene with an incompatible, irrational, outcome. In a study on emotion processing Hepach and Westermann (2013) presented 10- and 14-monthold infants with scenes in which adults were performing actions that were either congruent (happy and patting) or incongruent (happy and thumping) with their emotional expression. Despite no differences in looking times, the 14-month-olds, but not the 10-month-olds showed increased pupil dilation when watching the incongruent scenes (happy and thumping; angry and patting). Importantly, apart from suggesting greater sensitivity of pupil dilation than looking time measures (Jackson \& Sirois, 2009; Geangu, 2011; Hepach \& Westermann, 2013), the studies by Gredebäck 
\& Melidner (2010) and Hepach \& Westermann (2013) also illustrate the potential to study subtle changes in development across different age groups (see also Addyman, Rocha, \& Mareschal, 2014; Chatham, Frank, \& Munakata, 2009; Geangu et al., 2011; Morita, Slaughter, Katayama, Kitazaki, Kakigi, \& Itakura, 2012; Verschoor, Paulus, Spapé, Biro, \& Hommel, 2015).

Other studies have combined pupil size measures with measures of visual scan patterns (Anderson et al., 2006; Falck-Ytter, 2008; Morita et al., 2012; Nuske, Vivanti, \& Dissanayake, 2015), and fixation patterns (Addyman, Rocha, \& Mareschal, 2014). This work has served to study links between reactive (involuntary) measures and predictive (voluntary) measures of infant attention where infants shift their gaze to anticipate the outcome of an action (e.g., Johnson, Amso, \& Slemmer, 2003; McMurray \& Aslin, 2004).

Some studies have addressed pupil dilation as an indicator of children's emotional arousal in response to social stimuli (e.g., Martineau et al., 2011; Geangu et al., 2011; Nuske, Vivanti, \& Dissanayake, 2014; Nuske et al., 2015) and have integrated pupil size measures with behavioral paradigms to explore the underlying motivation of behavior (Hepach et al., 2012). In one study, two-year-old children showed similar levels of pupil dilation when they themselves helped or a third party helped someone in need, and both differed from when the needy person received no help (Hepach et al., 2012). This result suggested that children were not motivated to help in order to "get credit" for their helping but suggested that their motivation lay in the person in need be helped. The more children's pupil dilation increased to the need situation, the faster they were to initiate their helping behavior (Hepach et al., 2013). Measures of pupil dilation have proven fruitful in various research areas of developmental psychology and in the following we cover some of the techniques have 
been proposed to design and analyze pupillometry studies with young children.

Integrating measures of pupil dilation into studies with infants and toddlers poses a set of challenges regarding the design of the study as well as the properties of the employed stimuli. Given the sensitivity of pupil size to light (resulting in involuntary constriction) it is imperative that the overall luminance of stimuli is controlled for and that there are no systematic differences in luminosity between experimental conditions.

\section{Practical Considerations for Infant Studies with Pupil Dilation}

A central challenge in research with infants is the participants' short attention span and lack of cooperation. Pupillometry is no different and paradigms have to be designed and analyzed under this constraint. In the following, we propose that there are ways to obtain clean estimates of pupil dilation when considering several important points. In an ideal case, the eye tracker receives a continuous signal of pupil size while participants remain focused on the presentation screen without changes in luminosity. However, this ideal case is almost never the reality and different considerations enter the study design, data acquisition, and analysis process (see Table 2 for an overview).

\section{Study Set-up}

The lighting of the room should be kept constant for all participants throughout the study and the computer screen should have the same brightness/contrast levels for all participants. Importantly, some computer screens illuminate the surface unevenly (because the light source is on one side of the screen only) and may be brighter on one side than the other; these are best avoided (see also Aslin, 2012). An important consideration is that the stimuli on which pupil dilation is measured should not vary systematically in their luminance between experimental 
conditions as this would introduce confounds into the study design. Ideally all stimuli should be equated for luminance (e.g., by using Photoshop). However, sometimes this is not possible, for example when using video clips that vary in luminosity over time. In these cases it should be considered whether any obtained effects could be explained merely on the basis of luminosity differences (see Gredebäck \& Melinder (2010) for an example of how the pattern of pupil dilation results was opposite to that predicted by the luminance of the stimuli).

There is currently no gold standard on how to process and analyze pupil diameter data and on how to deal with noisy data (see Table 3 for an overview). Two main sources of data loss are eye blinks and look-aways from the screen (Fig. 3).

Gaps in pupil measurements during short eye blinks can be interpolated. However, look-aways pose more difficulty. In a typical experimental setup where the screen is brighter than the rest of the room, every look-away results in an increase in pupil size (adaptation to darker environment) and every re-focusing to the computer screen leads to a light reflex and pupillary constriction. This component can have a considerable influence on measures of pupil diameter over time. The presentation of stimuli on the computer screen should therefore be attractive enough to hold participants' attention as much as possible during the experimental manipulation. In effect the aim of pupil dilation studies is opposite to looking time paradigms: pupil dilation studies work best when the infant looks at the screen as much as possible, whereas looking time studies rely on infants looking away from the stimulus.

\section{Data Processing and Analyses}

The majority of eye trackers collect data for both eyes. Values for the size of the left and the right pupil are collected at the sampling frequency of the eye tracker, e.g., $60 \mathrm{~Hz}$. Several steps are necessary to reduce the noise of the raw data provided 
by the eye tracking system. These include the pre-processing of the data (filtering and interpolation) and transformation (e.g., baseline-correction) before statistical analyses can be carried out. An overview of the various analyses steps involved as well as examples from published work are provided in Table 2.

Data for each eye should be filtered to remove extreme sample-to-sample differences. Filters will differ with respect to the amount of data they remove. To investigate the effects of filtering it can be useful to plot the pupil data for each participant and for each eye (see Fig. 4). For further analysis, there should be no spikes but rather a continuous curve of data points (see Fig. 2A). The pupil size data will contain gaps for periods where the eye tracker did not capture the participant's eyes. Eye blinks will cause brief interruptions of eye capture, but loss of tracking due to head movements or look-aways can last much longer. An important consideration is the treatment of the resulting missing data and several approaches have been suggested, e.g., discarding trials with too few data points (see Table 3). The filtered and interpolated data can now be averaged to arrive at a composite measure of pupil diameter change or be transformed into cubic splines to retain the dynamic nature of the time series data (see Table 3 and see Nyström et al., for an online resource to analyze time-series data).

To investigate psychological influences on pupil diameter researchers timelock analysis to the time point at which an experimental manipulation (e.g., presentation of a picture; occurrence of an unexpected event in a video) occurred. The assumption is that pupil diameter will increase after the manipulation depending on experimental condition and different proposals have been put forward to analyze such changes in pupil diameter (see Table 2 for details). Given that the baseline pupil size for each participant can vary between participants and trials, a common approach is to 
baseline-correct all values. For this purpose, pupil size during a short time interval (e.g., $300 \mathrm{~ms}$ ) before the onset of the experimental manipulation is averaged to form the baseline. This value is then subtracted from the measured pupil size after the experimental manipulation. It is best not to include components of PC and PR in the baseline interval. For each participant the global average curve across several trials will result in the typical mean phasic changes in pupil dilation (see Fig. 2E). When analyzing tonic changes the pupil diameter is averaged within a specified time interval and statistically compared between experimental conditions (see Table 3 for details).

\section{Conclusions and Future Directions}

For infants and toddlers the world is more than a 'blooming and buzzing confusion' (Rakison \& Oakes, 2008). Infants actively explore their environment to learn from both the physical and social world around them. In this sense (socio-) cognitive development is tied to the processing of novel information as concepts and representations are formed (Westermann, Mareschal, Johnson, Sirois, Spratling, \& Thomas, 2007). Pupillometry offers a novel means to capture this processing. In contrast to looking time, pupil dilation does not suffer from a diminishing response over trials when participants become tired and less attentive because it does not require them to look at a stimulus for more than a few seconds (Jackson \& Sirois, 2009; see also Sirois \& Jackson, 2012, for a detailed discussion). Several studies have found measures of pupil dilation to be more sensitive to differences in experimental conditions than measures of looking time (Geangu et al., 2011; Jackson \& Sirois 2009; Hepach \& Westermann, 2013). In pupillometry, experimental designs are dynamic and pupillary changes can be assessed over brief time periods of time, too 
short to differentiate between experimental conditions with measures of looking times (Hepach \& Westermann, 2013).

By far the greatest appeal of pupillometry is its potential to address question above and beyond those investigated in looking time studies. Time-locked pupillary changes to critical events can pinpoint the onset of processing (Sirois \& Jackson, 2012). In addition, pupil dilation is a physiological measure that first and foremost assesses internal arousal. This provides a means to study infants' and toddlers' emotional processing also in comparison to atypical populations (e.g., Nuske et al., 2014, 2015). Indeed little is known about how infants process specific emotions and how this is related to their own emotional response. Furthermore, changes in children's internal arousal can reveal motivational states which opens up the possibility to study the underlying mechanisms of not only prosocial but behavior more generally (Hepach et al., 2012, 2015; see also, Hepach, Vaish, \& Tomasello, 2013).

As is the case for any emerging paradigm in research there are challenges that lie ahead. First, it is important to systematically relate changes in pupil diameter to other established physiological measures to clarify whether increased pupil dilation reflects increases in sympathetic nervous system activity similar to increased skin conductance or heart rate acceleration as opposed to heart rate deceleration (Richards \& Casey, 1991). Another possibility is that changes in pupil size are more reflective of decreases in heart rate variability as they have been documented in cases where children are in states of increased attention. Of central importance, however, is the methodological challenge of developing a standard in analyzing pupil diameter data similar to approaches in infant eye tracking (e.g., Oakes, 2012) and ERP (e.g., Stets, Stahl, \& Reid, 2012). With anticipated increase in pupillometry studies some 
approaches will prove to be more robust and replicable than others. This will help researchers to establish a standard procedure not just for analyzing pupil dilation data but also to cope with additional sources of noise such as the effects of eye movements (Brisson, Mainville, Mailloux, Beaulieu, Serres, \& Sirois, 2013) and prior light exposure (Szabó, Tokaji, Kálmán, Oroszi, Pestenácz, \& Janka, 2004), and potential systematic luminance differences between conditions on pupil dilation (Aslin, 2012).

A further challenge is to clarify the psychological nature of pupil dilation. The human pupil dilates in different contexts suggesting that there is no single psychological process. Given that psycho-sensory stimulation reflects both affective and cognitive factors, it is most helpful to conceive of pupil dilation as a composite marker of psychological engagement. In this sense future work will need to clarify the nature of the psychological processes that reliably dilate the pupils. One interesting future direction in infant pupillometry is a combination of anticipatory looking and pupil dilation measures to assess both mere responses to novelty (increased pupil dilation but not anticipation) and violations of prior held expectations (increased pupil dilation and anticipation).

In sum, pupillometry in preverbal or just-verbal populations provides researchers with a non-invasive tool to be combined with other measures in eye tracking research (Gredebäck et al., 2009; Oakes, 2012). The appeal of pupil dilation is that it provides a continuous measure of sympathetic activity that is enhanced both in emotionally charged situations, while exerting mental effort and in states of surprise. In this sense, pupillometry is a new scientific lens through which otherwise unobservable process become visible, quantifiable, and interpretable. This opens up the possibility to study genuinely new questions about human development from its beginning in infancy. 


\section{References}

Addyman, C., Rocha, S., \& Mareschal, D. (2014). Mapping the origins of time: Scalar errors in infant time estimation. Developmental Psychology, 50(8), 2030.

Anderson, C. J., Colombo, J., \& Shaddy, D. J. (2006). Visual scanning and pupillary responses in young children with autism spectrum disorder. Journal of Clinical and Experimental Neuropsychology, 28(7), 1238-1256.

Aslin, R. N. (2007). What's in a look? Developmental Science, 10, 48-53.

Aslin, R. N. (2012). Infant eyes: A window on cognitive development. Infancy, 17(1), 126-140.

Beatty, J., \& Lucero-Wagoner, B. (2000). The pupillary system. In J.T. Cacioppo, L.G. Tassinary, \& G.G. Berntson (Eds.), Handbook of psychophysiology (2nd ed., pp. 142-162). New York: Cambridge University Press.

Bradley, M. M., Miccoli, L., Escrig, M. A., \& Lang, P. J. (2008). The pupil as a measure of emotional arousal and autonomic activation. Psychophysiology, 45(4), 602-607.

Bradshaw, J. (1967). Pupil size as a measure of arousal during information processing, Nature, 216, 515-516.

Brisson, J., Mainville, M., Mailloux, D., Beaulieu, C., Serres, J., \& Sirois, S. (2013). Pupil diameter measurement errors as a function of gaze direction in corneal reflection eyetrackers. Behavior Research Methods, 45(4), 1322-1331.

Cashon, C. H., \& Cohen, L. B. (2000). Eight-month-old infants' perception of possible and impossible events. Infancy, 1(4), 429-446.

Chatham, C. H., Frank, M. J., \& Munakata, Y. (2009). Pupillometric and behavioral markers of a developmental shift in the temporal dynamics of cognitive control. Proceedings of the National Academy of Sciences, 106(14), 5529-5533.

Cohen, L. B., DeLoache, 1. S., \& Pearl, R. (1977). An examination of interference effects 
in infants memory for faces. Child Development, 48, 88-96.

de Haan, M. (2013). Infant EEG and event-related potentials. Psychology Press.

Fantz, R. L. (1963). Pattern vision in newborn infants. Science, 140(3564), 296-297.

Fantz, R. L. (1964). Visual experience in infants: Decreased attention to familiar patterns relative to novel ones. Science, 146(3644), 668-670.

Falck-Ytter, T. (2008). Face inversion effects in autism: a combined looking time and pupillometric study. Autism Research, 1(5), 297-306.

Geangu, E., Hauf, P., Bhardwaj, R., \& Bentz, W. (2011). Infant pupil diameter changes in response to others' positive and negative emotions. PloS one, 6(11), e27132.

Goldwater, B. C. (1972). Psychological significance of pupillary movements. Psychological Bulletin, 77, 340-355.

Granholm, E., \& Steinhauer, S. R. (2004). Pupillometric measures of cognitive and emotional processes. International Journal of Psychophysiology, 52(1), 1-6.

Gredebäck, G., Johnson, S., \& von Hofsten, C. (2009). Eye tracking in infancy research. Developmental Neuropsychology, 35(1), 1-19.

Gredebäck, G., \& Melinder, A. (2010). Infants' understanding of everyday social interactions: a dual process account. Cognition, 114(2), 197-206.

Gredebäck, G., Eriksson, M., Schmitow, C., Laeng, B., \& Stenberg, G. (2012). Individual differences in face processing: Infants' scanning patterns and pupil dilations are influenced by the distribution of parental leave. Infancy, 17(1), 79-101.

Hakerem, G. (1974). Conceptual stimuli, pupillary dilation and evoked cortical potentials: a review of recent advances. In Pupillary Dynamics and Behavior (pp. 135-158). Springer US.

Hepach, R., Vaish, A., \& Tomasello, M. (2012). Young children are intrinsically motivated to see others helped. Psychological Science, 23(9), 967-972. 
Hepach, R., \& Westermann, G. (2013). Infants' sensitivity to the congruence of others' emotions and actions. Journal of Experimental Child Psychology, 115(1), 16-29.

Hepach, R., Vaish, A., \& Tomasello, M. (2013). A new look at children's prosocial motivation. Infancy, 18(1), 67-90.

Hepach, R., Vaish, A., \& Tomasello, M. (2015). Novel paradigms to measure variability of behavior in early childhood: posture, gaze, and pupil dilation. Frontiers in Psychology, 6.

Hess, E. H., \& Polt, J. M. (1960). Pupil size as related to interest value of visual stimuli. Science, 132, 349-350.

Hochmann, J. R., \& Papeo, L. (2014). The Invariance Problem in Infancy A Pupillometry Study. Psychological Science, 25(11), 2038-2046.

Jackson, I., \& Sirois, S. (2009). Infant cognition: going full factorial with pupil dilation. Developmental Science, 12(4), 670-679.

Johnson, S. P., Amso, D., \& Slemmer, J. A. (2003). Development of object concepts in infancy: Evidence for early learning in an eye-tracking paradigm. Proceedings of the National Academy of Sciences, 100(18), 10568-10573.

Kahneman, D., \& Beatty, J. (1966). Pupil diameter and load on memory. Science, 154, $1583-1585$.

Kahneman, D., Tursky, B., Shapiro, D., \& Crider, A. (1969). Pupillary, heart rate, and skin resistance changes during a mental task. Journal of Experimental Psychology, 79, $164-167$.

Kahneman, D. (1973). Attention and effort. Englewood Cliffs, NJ: Prentice-Hall. Laeng, B., Sirois, S., \& Gredebäck, G. (2012). Pupillometry A Window to the Preconscious? Perspectives on Psychological Science, 7(1), 18-27. 
Laeng, B., \& Sulutvedt, U. (2014). The eye pupil adjusts to imaginary light. Psychological science, 25(1), 188-197.

Libby, W. L., Lacey, B. C., \& Lacey, J. I. (1973). Pupillary and cardiac activity during visual attention. Psychophysiology, 10(3), 270-294.

Loewenfeld, I. (1958). Mechanisms of reflex dilatation of the pupil. Documenta Ophthalmologica, 12(1), 185-448.

Loewenfeld, I. E. (1993). The pupil: Anatomy, physiology, and clinical applications, Detroit, MI: Wayne State University Press.

Lowenstein, 0. (1920). Experimentelle Beiträge zur Lehre von den katatonischen Pupillenveränderungen. Monatsschrift fiir Psychatrie und Neurologie, 47, 194-215. Lowenstein, O., Feinberg, R. \& Loewenfeld, I. E. (1963). Pupillary movements during acute and chronic fatigue. Invest. Ophthalmol., 2, 138-157.

Marshall, S. P. (2002). The index of cognitive activity: Measuring cognitive workload. In Human factors and power plants, 2002. proceedings of the 2002 IEEE 7th conference on (pp. 7-5). IEEE.

Martineau, J., Hernandez, N., Hiebel, L., Roché, L., Metzger, A., \& Bonnet-Brilhault, F. (2011). Can pupil size and pupil responses during visual scanning contribute to the diagnosis of autism spectrum disorder in children?. Journal of Psychiatric Research, 45(8), 1077-1082.

Morita, T., Slaughter, V., Katayama, N., Kitazaki, M., Kakigi, R., \& Itakura, S. (2012). Infant and adult perceptions of possible and impossible body movements: An eyetracking study. Journal of Experimental Child Psychology, 113(3), 401-414.

Nieuwenhuis, S., De Geus, E. J., \& Aston-Jones, G. (2011). The anatomical and functional relationship between the $\mathrm{P} 3$ and autonomic components of the orienting response. Psychophysiology, 48, 162- 175. 
Nuske, H. J., Vivanti, G., \& Dissanayake, C. (2014). Brief report: Evidence for normative resting-state physiology in autism. Journal of Autism and Developmental Disorders, 44(8), 2057-2063.

Nuske, H. J., Vivanti, G., \& Dissanayake, C. (2015). No Evidence of Emotional Dysregulation or Aversion to Mutual Gaze in Preschoolers with Autism Spectrum Disorder: An Eye-Tracking Pupillometry Study. Journal of Autism and Developmental Disorders, 1-13.

Nyström, P., Falck-Ytter, T., \& Gredebäck, G. (2015). The TimeStudio Project: An open source scientific workflow system for the behavioral and brain sciences. Behavior Research Methods, 1-11.

Oakes, L. M. (2012). Advances in eye tracking in infancy research. Infancy, 17(1), 1-8.

Partala, T., \& Surakka, V. (2003). Pupil size variation as an indication of affective processing. International Journal of Human-Computer Studies, 59, 185-198.

Preuschoff, K., 't Hart, B. M., \& Einhäuser, W. (2011). Pupil dilation signals surprise: evidence for noradrenaline's role in decision making. Frontiers in Neuroscience, 5, 112.

Rakison, D. H., \& Oakes, L. M. (2008). Early category and concept development. Oxford University Press.

Richards, J. E., \& Casey, B. J. (1991). Heart rate variability during attention phases in young infants. Psychophysiology, 28(1), 43-53.

Sirois, S., \& Jackson, I. R. (2011). Pupil dilation and object permanence in infants. Infancy, 17(1), 61-78.

Sirois, S., \& Brisson, J. (2014). Pupillometry. Wiley Interdisciplinary Reviews: Cognitive Science, 5(6), 679-692.

Sokolov, E. N. (1963). Perception and the Conditioned Reflex. Oxford: Pergamon Press. 
Stets, M., Stahl, D., \& Reid, V. M. (2012). A meta-analysis investigating factors underlying attrition rates in infant ERP studies. Developmental Neuropsychology, $37(3), 226-252$.

Szabó, Z., Tokaji, Z., Kálmán, J., Oroszi, L., Pestenácz, A., \& Janka, Z. (2004). The effect of bright light exposure on pupillary fluctuations in healthy subjects. Journal of affective disorders, 78(2), 153-156.

Verschoor, S. A., Paulus, M., Spapé, M., Biro, S., \& Hommel, B. (2015). The developing cognitive substrate of sequential action control in 9-to 12-month-olds: Evidence for concurrent activation models. Cognition, 138, 64-78.

Westermann, G., Mareschal, D., Johnson, M. H., Sirois, S., Spratling, M. W., \& Thomas, M. S. (2007). Neuroconstructivism. Developmental Science, 10(1), 75-83.

Wilhelm, H. (1991). Pupillenreaktionen - Pupillenstörungen. Stuttgart: Kohlhammer. Woodward, A. L. (1998). Infants selectively encode the goal object of an actor's reach. Cognition, 69(1), 1-34. 
Table 1

Overview of studies investigating infants' and toddler's pupil diameter.

\begin{tabular}{|c|c|c|c|c|}
\hline Authors & Research domain & $\begin{array}{c}\text { Ages } \\
\text { studied }\end{array}$ & Pupillary changes & Research question details \\
\hline Addyman et al., (2014) & Learning & $\begin{array}{l}4 \mathrm{~m}, 6 \mathrm{~m}, \\
10 \mathrm{~m}, \& \\
14 \mathrm{~m}\end{array}$ & Phasic & Infants' learning in an interval timing task. \\
\hline Anderson et al., (2006) & Social cognition & 12 to $72 \mathrm{~m}$ & Tonic & Children's responses to face and non-face stimuli. \\
\hline Chatham et al., (2009) & Cognitive control & 3.5 and $8 \mathrm{y}$ & Phasic & Dissociating reactive and proactive context control. \\
\hline Jackson \& Sirois (2009) & Physical cognition & $8 \mathrm{~m}$ & Phasic & $\begin{array}{l}\text { Infants' responses to conceptually impossible and } \\
\text { perceptually novel events. }\end{array}$ \\
\hline Falck-Ytter (2008) & Social cognition & $4 \& 5 y$ & Phasic & $\begin{array}{l}\text { Pupillary changes while observing upright and inverted } \\
\text { face stimuli. }\end{array}$ \\
\hline $\begin{array}{l}\text { Gredebäck \& Melinder (2010; } \\
\text { 2011) }\end{array}$ & Social cognition & $\begin{array}{l}4 \mathrm{~m}, 6 \mathrm{~m} \& \\
12 \mathrm{~m}\end{array}$ & Phasic & $\begin{array}{l}\text { Infants' responses to irrational feeding events between } \\
\text { two adults. }\end{array}$ \\
\hline Gredebäck et al., (2012) & Social cognition & $14 \mathrm{~m}$ & Phasic & $\begin{array}{l}\text { Responses to pictures of caretakers and strangers } \\
\text { depending on maternal and paternal care. }\end{array}$ \\
\hline Geangu et al. (2011) & Social cognition & $6 \& 12 \mathrm{~m}$ & Tonic & Infants' responses to the emotional display of peers. \\
\hline Hepach et al. $(2012,2013)$ & Motivation & $24 \mathrm{~m}$ & Tonic & $\begin{array}{l}\text { Changes in children's pupil dilation after helping or not } \\
\text { helping an adult. }\end{array}$ \\
\hline Hepach \& Westermann (2013) & Social cognition & $10 \& 14 \mathrm{~m}$ & Phasic & $\begin{array}{l}\text { Infants' responses to congruence of adults' emotions and } \\
\text { actions. }\end{array}$ \\
\hline Morita et al., (2012) & Social cognition & $9 \& 12 \mathrm{~m}$ & Phasic & $\begin{array}{l}\text { Do infants show greater pupil dilation to impossible } \\
\text { compared to possible arm movements? }\end{array}$ \\
\hline Nuske et al. (2014) & Psychophysiology & $4 y$ & Tonic & $\begin{array}{l}\text { Baseline pupil diameter in children with ASD and } \\
\text { typically developing children. }\end{array}$ \\
\hline Nuske et al. (2015) & Social cognition & $4 y$ & Phasic & Young children's responses to averted and mutual gaze. \\
\hline Nyström et al., (2015) & Psychophysiology & $10 \mathrm{~m}$ & Pupillary light reflex & $\begin{array}{l}\text { Pupillary light reflex in infants at risk for developing } \\
\text { autism. }\end{array}$ \\
\hline Sirois \& Jackson (2011) & Physical cognition & $10 \mathrm{~m}$ & Phasic & Infants' responses to violations of object permanence. \\
\hline Verschoor et al. (2015) & Action control & $9 \& 12 \mathrm{~m}$ & Phasic & Infants' acquisition of sequential actions. \\
\hline
\end{tabular}


Note: This is a brief overview to illustrate the different research areas in which measures of pupil dilation have been applied.

Table 2

Several possibilities of experimental designs employing pupil dilation as a dependent measure.

Stimulus type

Still pictures

\section{Description}

Pupillary changes are measured in

response to still images, e.g., of

different emotional expressions

(Bradley et al., 2008)
Challenges

Pupils will accommodate to the varying

luminance of stimuli which will cause

pupillary constriction and dilation

irrespective of the image's content.

Some presentation software programs need time to load images resulting in a brief blank screen in between stimuli,

leading to luminance-dependent changes in pupil size.

\section{Recommendations}

Ideally, the luminance of all stimuli should be the same. If multiple images of the same category are used, e.g., samples of emotionally neutral compared to emotionally arousing images, it should be made sure that the average luminance does not differ between sets of stimuli (see, Bradley et al., 2008, for an example).

Present a neutral image of the same luminance as the target stimulus, e.g., a grey image, before the actual target to control for pupillary changes due to luminance.

Combine images in a video to avoid black blanks between the presentation of images.

Time-lock pupillary changes after pupillary constriction (PC) and pupillary re-dilation (PR) with the onset of pupillary oscillation (PO).

Present an attention grabbing sequence to fixate participants' gaze on the screen. Then present the stimulus.

neutral image on the screen while they

listen to the stimulus.

may disengage from the presentation surface focussing on other aspects of the room causing pupillary changes due to the room luminance.

Use the same stimulus while playing different stimuli sounds. 
Video

A scene is presented as a video making the stimulus presentation engaging for infants and young children. The experimental manipulation occurs during the video sequence.

The stimulus itself is not part of the experimental manipulation. The trigger occurs before the measurement stimulus or in between two neutral stimuli.
The pupils are constantly adapting to the physical properties of the video,

potentially obscuring the psychological effects on pupil dilation.
If video recordings are used, ensure that the lighting conditions were the same when filming the different scenarios.

Time-lock changes in pupil dilation to the event in the video. A pre-defined period before the event serves as a baseline which is subtracted from all succeeding values.

Focus participants' attention to the video before the actual manipulation occurs, e.g., through an attention-grabbing event in the video.

Measure pupil dilation from a still-frame of the video scene introduced after the manipulation event to reduce noise.

The effect resulting from the experimental manipulation before the neutral stimulus has to be significant enough to 'carry over' to the moment of measurement.

Measure pupil diameter over a period of several seconds time-locked to the onset of the stimulus (see, Hepach et al. 2012, for an example of using neutral pictures and videos)
The stimulus itself should not be too distracting and engaging. 
Table 3

Crucial steps in processing, transforming, and analyzing pupil diameter. Publications are provided as examples to each step.

\begin{tabular}{|c|c|c|}
\hline Key Analysis Aspects & Specifications & References \\
\hline \multicolumn{3}{|l|}{ Filter to remove extreme values } \\
\hline \multirow{2}{*}{ Stimulus during measurement } & Digital low-pass & Addyman et al., (2014); Geangu et al. \\
\hline & Picture or photograph & (201d). Jackson \& S Sirois 2009 . Sirois \& \\
\hline \multirow[t]{3}{*}{ Time point of measurement (duration in s) } & Moving average & 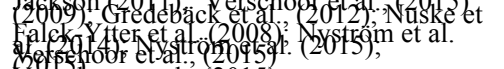 \\
\hline & Quantile cut-off & 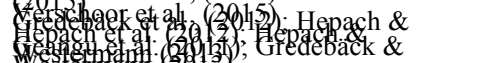 \\
\hline & $\begin{array}{l}\text { Savitzky-Golay filter } \\
\text { Standard deviation cut-off }\end{array}$ & 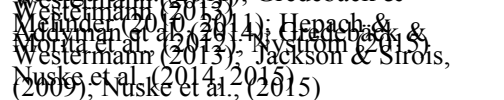 \\
\hline \multirow{3}{*}{ Procedure to fill gaps between samples } & Computer animation & Addyman et al. (2014); Hepach et al. \\
\hline & Linear interpolation for each eye data & 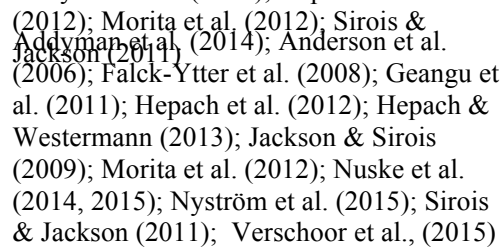 \\
\hline & $\begin{array}{l}\text { Linear interpolation between data for both } \\
\text { eyes }\end{array}$ & $\begin{array}{l}\text { Geangu et al. (2011); Jackson \& Sirois } \\
\text { (2009); Sirois \& Jackson (2011) }\end{array}$ \\
\hline & Cubic splines & $\begin{array}{l}\text { Addyman et al. (2014); Geangu et al. } \\
\text { (2011); Jackson \& Sirois (2009); Sirois \& } \\
\text { Jackson (2011) }\end{array}$ \\
\hline & Trial or participant filter according to criterion & $\begin{array}{l}\text { Falck-Ytter et al. (2008); Gredebäck \& } \\
\text { Melinder (2011); Gredebäck et al., (2012); } \\
\text { Nyström et al. (2015) }\end{array}$ \\
\hline & Replacing extreme values with group mean & $\begin{array}{l}\text { Gredebäck \& Melinder (2011); Gredebäck } \\
\text { et al., (2012) }\end{array}$ \\
\hline & \multirow{2}{*}{$\begin{array}{l}\text { Binning of data } \\
\text { Data resampling }\end{array}$} & Addyman et al. (2014) \\
\hline & & Nyström et al. (2015) \\
\hline
\end{tabular}


Statistical analysis

Baseline period

Dependent measure
During experimental manipulation

Analysis of Variance (ANOVA)

No experimental manipulation

None

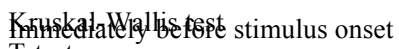
T-test

Likelihood-ratio test

Linear mixed modelling

Repeated measurement GLM

$15 \mathrm{~s}$ before

After the first data point

Overall mean per participant

Functional pupil diameter

Baseline-corrected mean change

Latency of the pupillary light reflex

Coefficient of variation in pupil diameter;

Change from each participant's grand mean

Pupillary inversion sensitivity measure

Time between peaks in pupil dilation

Mean pupil diameter
Melinder (2010, 2011)

Chatham et al. (2009)

Hepach et al. (2012)

Morita et al. (2012)

Nuske et al. (2015)

Sirois \& Jackson (2011)

Anderson et al. (2006)

Geangu et al. (2011)

(2009)

Ner

Nusket dR.(20); 4 Jredeback et al., (2012)

(2)

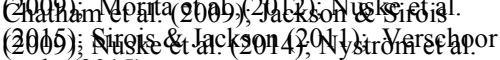

(2)

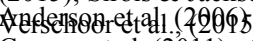

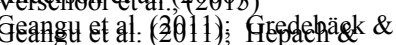

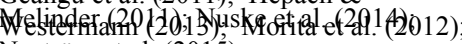

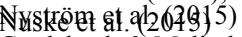

Gredebäkck Melinder (2010) 2011)

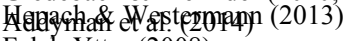

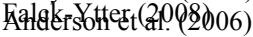

Hepach et al., (2012)

Falck-Ytter (2008)

Gredebäck et al., (2012)

Jackson \& Sirois (2009); Geangu et al.

(2011); Sirois \& Jackson (2011)

Anderson et al. (2006); Geangu et al.

(2011); Gredebäck \& Melinder (2010);

Gredebäck et al., (2012); Hepach et al.

(2012); Hepach \& Westermann (2013);

Morita et al. (2012); Nuske et al. (2015);

Verschoor et al., (2015)

Nyström et al. (2015)

Chatham et al. (2009)

Falck-Ytter (2008)

Addyman et al. (2014)

Nuske et al. (2014) 


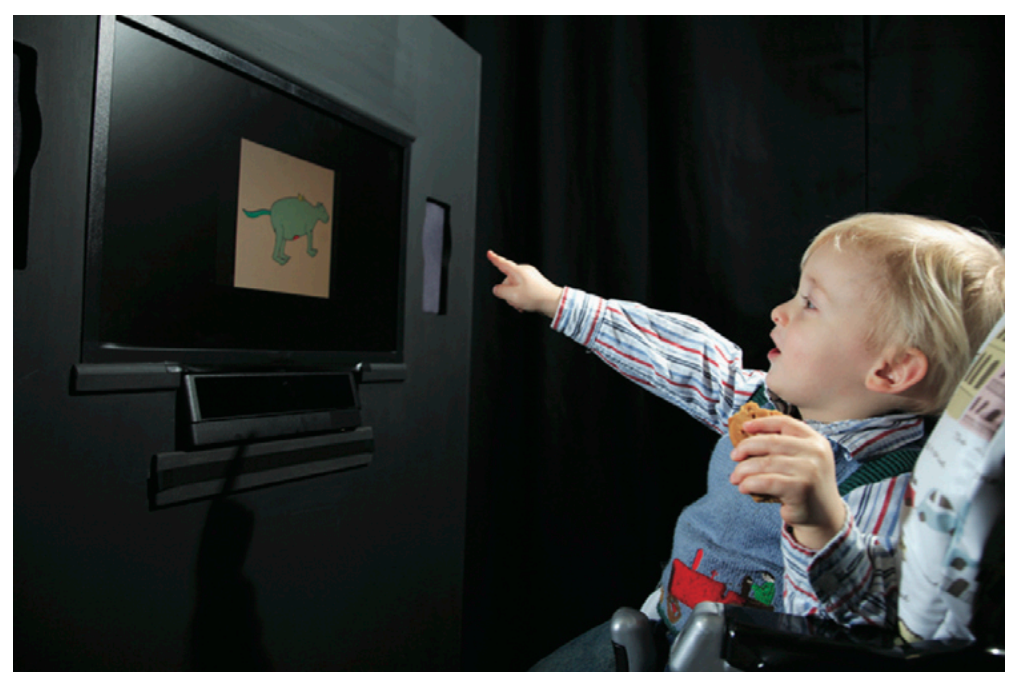

Figure 1. An illustration of an eye tracker set-up. 
A
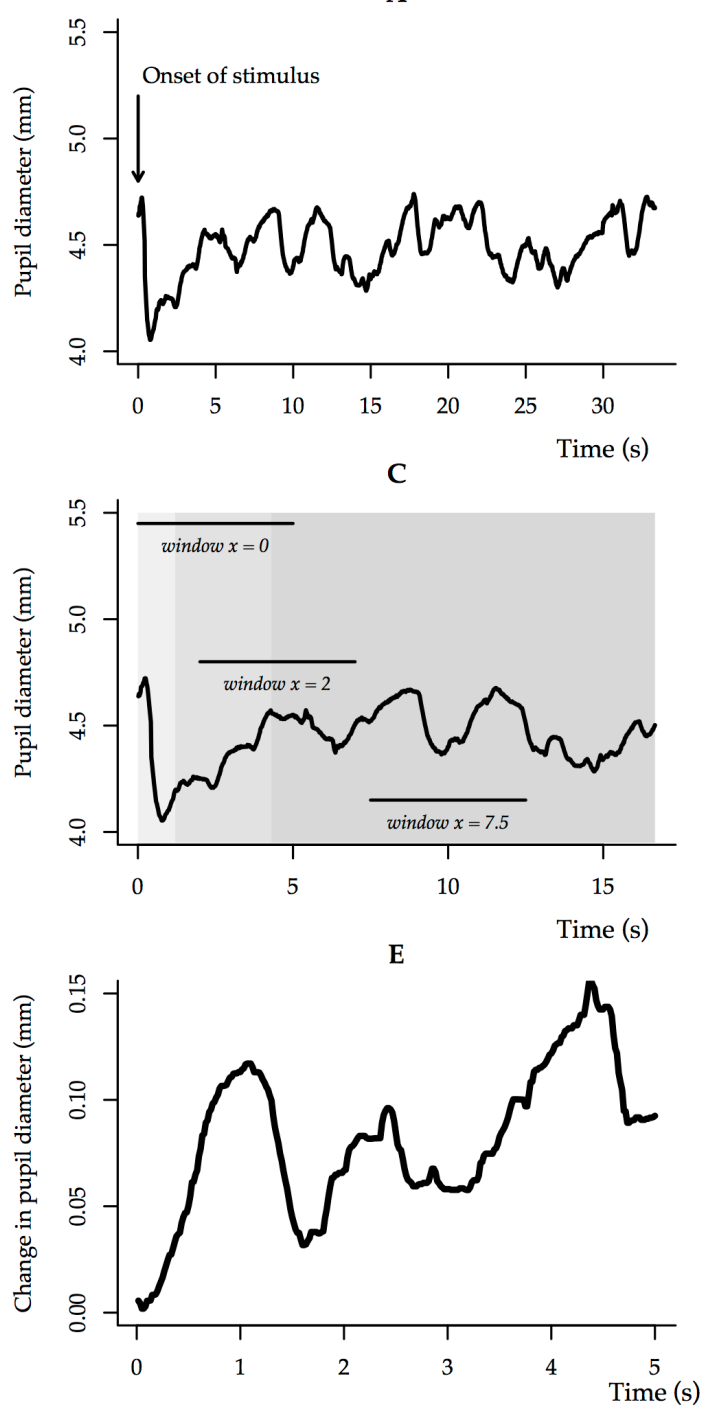

B

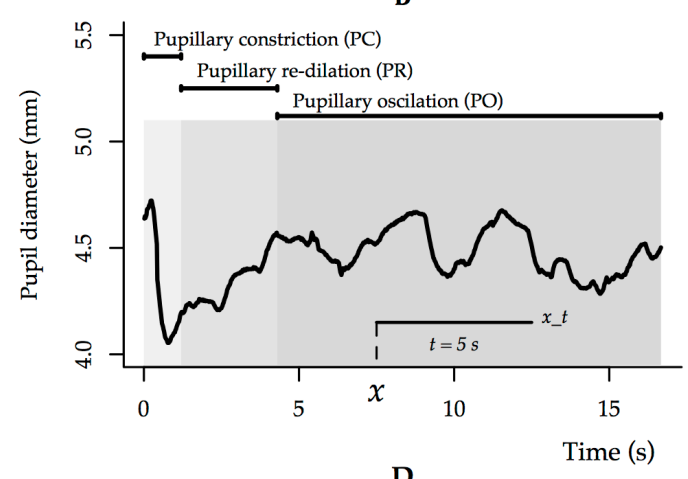

D

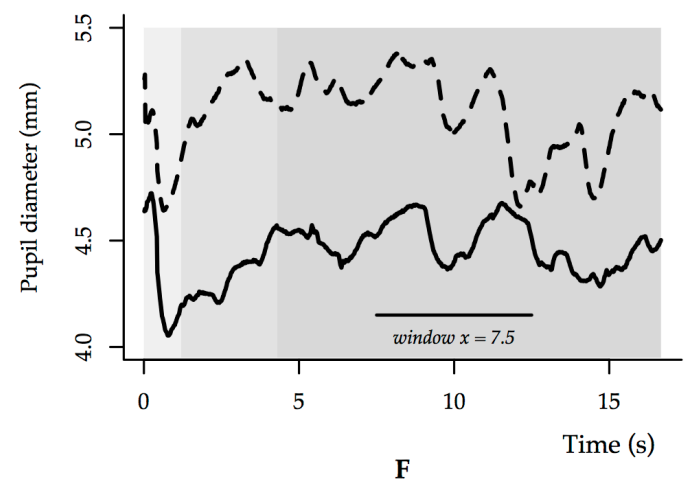

$\mathbf{F}$

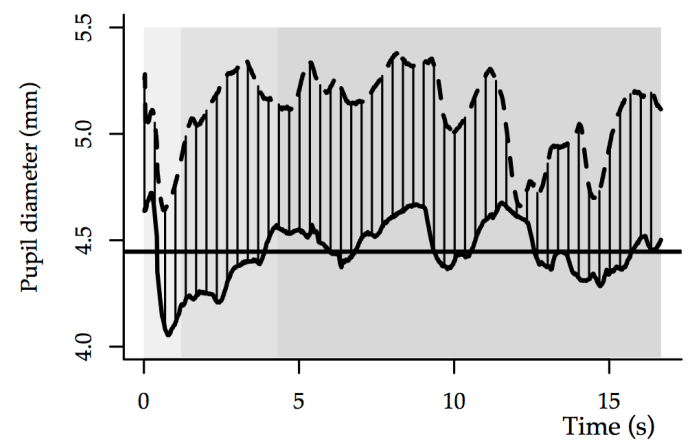

Figure 2. Several aspects of the pupillary signal. The x-axis denotes times in seconds. The y-axis denotes the pupil diameter. $A$ The typical pattern of the change in pupil size following the onset of a light source, e.g., a stimulus on the computer screen. $B$ The different components of the pupillary signal with an example time window starting at time point $\mathrm{x} . C$ Three different time windows of the same length but at different time points. The average of each window would vary even though all have the same length. $D$ Two pupillary curves following the onset of the same stimulus. Even though the same stimulus was shown, the detailed pattern of both curves is different. $E$ An example of phasic change in pupil diameter. The values are baseline- 
corrected and therefore the pupil size is ' 0 ' at the beginning of the curve. $F$ An example of the difference of two pupillary curves. The dashed vertical lines illustrate the difference. 


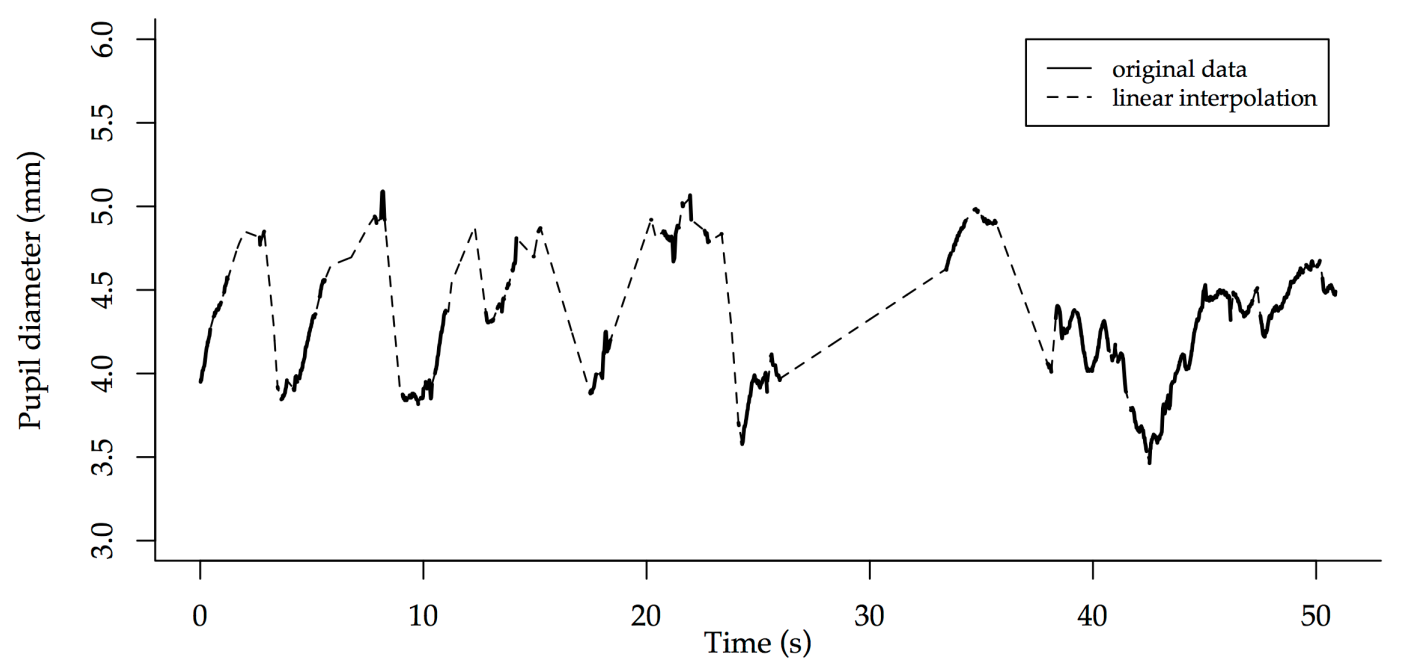

Figure 3. The time course of pupil dilation (solid line) with the superimposed output of a linear interpolation algorithm to fill all the gaps in data. Linear interpolation becomes problematic if the gaps are too large and likely result from participants looking away from the computer screen. 


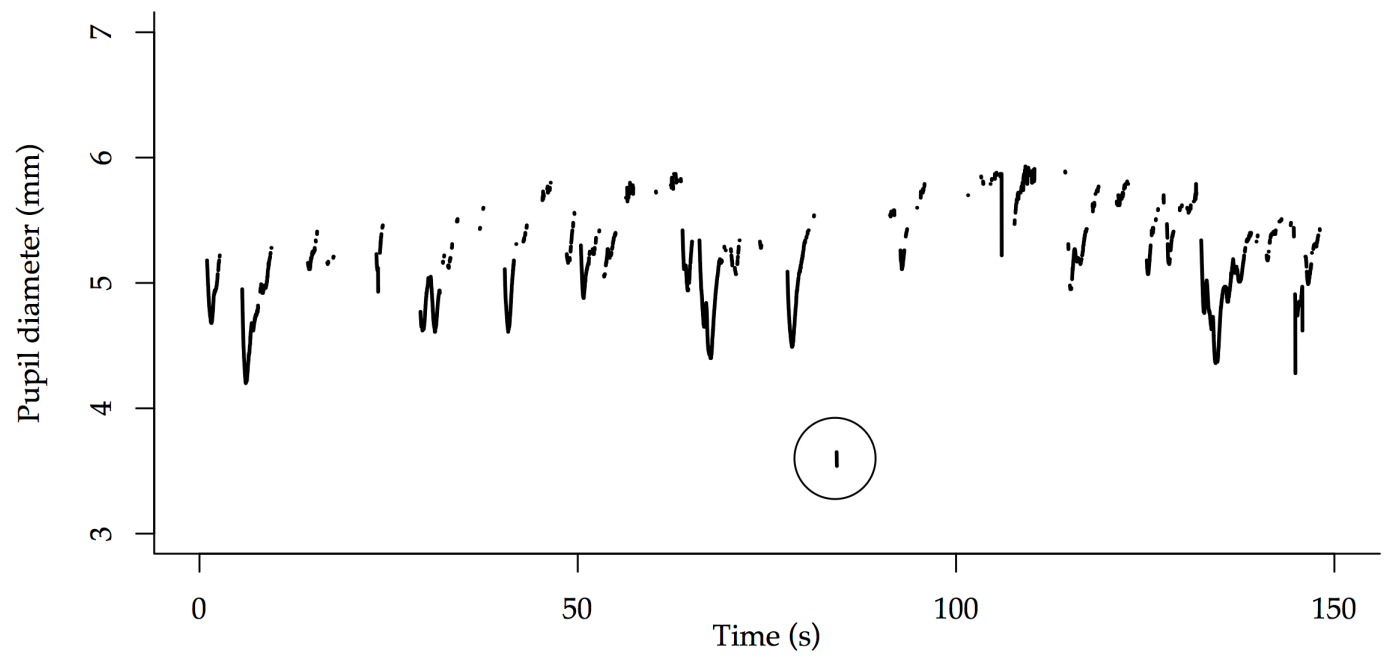

Figure 4. An example of a pupillary curve with an outlying sample (marked with the circle) which needs to be filtered out to avoid artifacts in the data set. 